\title{
Evolution de la composition corporelle des jeunes bovins mâles entiers de race Limousine entre 9 et 19 mois.
}

\section{I. - Composition anatomique}

\author{
J. ROBELIN, Y. GEAY et C. BERANGER
}

avec la collaboration technique de Roland JAIL, $L_{,} R$ et Robert JAIL, LER

Laboratoive de la Production de Viande

Centre de Recherches de Clermont-Ferrand, I.N.R.A., Theix, Saint-Genès Champanelle, 63Iro Beaumont (France)

\section{Résumé}

L'évolution de la composition anatomique de jeunes bovins mâles de race Limousine entre 9 et 19 mois (300 à $650 \mathrm{~kg}$ de poids vif) est décrite grâce à la méthode des abattages (5 animaux abattus et disséqués à 4 stades). Cette évolution est exprimée selon la relation allométrique (coefficient d'allométrie : $b$ ).

On retrouve la hiérarchie classique dans la croissance du squelette $(b=0,74)$, des muscles $(b=\mathrm{T}, \mathrm{OI})$ et de l'ensemble des dépotts adipeux $(b$ "moyen " = 1,59). Cependant, la croissance relative de ces derniers augmente entre 300 et $650 \mathrm{~kg}$ de poids vif de I, 25 à $2, \mathrm{I} 5$.

Ce sont les dépôts adipeux sous-cutanés qui ont la croissance relative la plus élevée $(b=2,44)$ suivis par les dépôts péritonéaux et périrénaux $(b=\mathrm{r}, 80)$, enfin par les dépôts intermusculaires et mésentériques $(b=\mathbf{r}, 48)$.

Les masses musculaires du dos et du nembre postérieur ont une croissance relative plus lente que celle de l'ensemble de la musculature.

Les particularités des jeunes bovins de race Limousine sont discutées en relation avec les données antérieures : état d'engraissement, répartition de la musculature et des dépôts adipeux.

\section{Introduction}

Nos conceptions sur la croissance et le développement des bovins reposent essentiellement sur des résultats obtenus avec des animaux castrés de race Angus ou Hereford, à développement musculaire faible (TROWBRIDGE, Mous'Ton et Haigh, igig; Callow, i948; Butterfield i i963; Tul, I966; SEEBECK, I967). 
Une étude que nous avons réalisée avec des animaux mâles entiers (RobELIN, GEAY et BÉRANGER, I974), et plus récemment les travaux d'ANDERSEx (I975) ont permis de mettre en évidence les principales particularités dư développement de ce type d'animal. 'Toutefois, ces travaux ont été effectués avec des animaux de races précoces (Frisonne ou Rouge Danoise), dont le potentiel de croissance musculaire est encore faible par rapport à celui des races à viande utilisées en France C'est pourquoi dans le cadre d'une étude de la production de viande à partir d'animaux de race Limousine (I), nous avons effectué une description détaillée de la croissance des jeunes taureaux de cette race à potentiel de croissance musculaire élevé, en insistant particulièrement sur la croissance des différentes régions musculaires et des différents types de dépôts adipeux au cours de la période d'engraissement entre 9 et 19 mois.

\section{Matériel et méthodes}

\section{Stades d'abattage}

Nous avons mesuré l'évolution de la composition corporelle des animaux par 1a méthode des abattages comparatifs. Nous avons abattu au total 20 animaux à 4 stades compris entre le sevrage ( 9 mois; poids vif voisin de $300 \mathrm{~kg}$ ) et un âge qui apparaissait maximal pour la production de jeunes bovins à croissance intensive (I9 mois; poids vif voisin de $700 \mathrm{~kg}$ ). Les deux stades intermédiaires ont été choisis de façon à diviser la période étudiée en sous-périodes de durée voisine, mais également sur la base de considérations zootechniques: d'une part, à l'âge de I3 mois (poids vif voisin de $450 \mathrm{~kg}$ ) correspondant en moyenne à celui d'un type de production très répandu dans la race Limousine (veaux de Lyon); d'autre part, à l'âge de 16 mois (poids vif voisin de $550 \mathrm{~kg}$ ) correspondant à l'âge moyen d'abattage des taurillons en France.

A chaque stade d'abattage, les animaux devant être abattus ont été choisis sur la base de leur poids vif et de leur âge, de façon à ce qu'ils soient représentatifs de l'ensemble de la population.

Les mesures effectuées à l'abattage (poids vif vide, poids des différents éléments du cinquième quartier, dissection de la carcasse, répartition des muscles selon leur localisation) ont été définis précédemment (ROBELIN, GEAY et BÉRANGER, I974; RoBELIN et GEAY, I976). En outre, nous avons séparé chacun des dépôts adipeux sous-cutanés et intermusculaires en trois parties (membre antérieur, membre postérieur et tronc) selon des repères anatomiques précis, correspondant aux limites définies pour les régions de la musculature (ROBELIN et GEAY, I976).

\section{Conduite des animaux}

Les animaux utilisés dans cette étude ont été achetés au sevrage à 9 mois, dans des élevages où ils avaient été pesés à différents âges et choisis en liaison avec une commission d'éleveurs, afin d'être représentatifs des animaux "moyens" de la race. Ils avaient été alimentés, en liberté au pâturage, au lait de leur mère

(r) Action concertée avec la Station de Recherches sur la Viande, financée par la Rénovation Rurale. 
et à l'herbe jusqu'à l'âge de 9 mois. Leur poids vif variait à cet âge entre 275 et $310 \mathrm{~kg}$.

Au cours de la période expérimentale, les animaux ont ensuite reçu à volonté, individuellement, en stabilisation entravée, une ration comportant $73 \mathrm{p}$. cent d'aliment concentré, 20 p. cent de luzerne déshydratée (ces deux aliments étant condensés) et $7 \mathrm{p}$. cent de foin de luzerne, afin de réaliser entre 9 et $\mathrm{I} 9$ mois une croissance pondérale voisine de leur potentiel maximal.

\section{Analyse des résultats}

Nous avons utilisé une relation logarithmique du second degré pour décrire l'évolution du poids $(\mathrm{Y})$ des différentes parties de l'organisme, par rapport aut poids vif vide $(\mathrm{X})$ :

$$
\log \mathrm{Y}=b_{0}+b_{1} \log \mathrm{X}+b_{2}(\log \mathrm{X})^{2}
$$

Ce modèle permet de tenir compte de l'évolution de la croissance relative de Y par rapport à $\mathrm{X}$. Il peut être considéré comme une extension de la ration allométrique classique :

$$
\text { Loy } \mathrm{Y}=b_{0}+b_{1} \log \mathrm{X}
$$

En effet, le rapport $b$ entre les taux de croissance de $\mathrm{Y}$ et celui de $\mathrm{X}$ : $\frac{d \mathrm{Y}}{\mathrm{Y}} \mid \frac{d \mathrm{X}}{\mathrm{X}}$ soit $\frac{d \log \mathrm{Y}}{d \log \mathrm{X}}$ est égal d'après l'équation (I) à :

$$
b=b_{1}+2 b_{2} \log \mathrm{X}
$$

Lorsque $b_{2}$ n'est pas significativement différent de o, la relation (I) devient linéaire et $b$ coefficient d'allométrie au sens strict est égal à $b_{1}$. Dans le cas contraire $\left(b_{2} \neq 0\right)$, le coefficient de croissance relative $b$ tiré de l'équation (3) peut être assimilé à un coefficient d'allométrie " instantané ». Nous avons donné à $b_{2}$ dans les tableaux la valeur 0 , lorsqu'elle n'était pas significativement différente de 0 au seuil $\mathrm{P}<\mathrm{O}, \mathrm{OI}$.

Dans le cas où $b_{2}$ était différent de o, nous avons toutefois calculé à partir de l'équation (3) un coefficient $b$ que nous avons appelé "moyen ", correspondant à la valeur moyenne de $X$. Ce coefficient n'a pas de signification statistique, mais il permet toutefois de comparer les éléments dont le coefficient de croissance relative varie, à ceux pour lesquels il demeure constant.

Nous avons utilisé le même modèle (I) pour décrire l'évolution du poids des différentes régions musculaires et des principaux muscles par rapport à la musculature totale, celle du poids de différents os par rapport au squelette, et celle des différents dépôts adipeux par rapport à l'ensemble de ces dépôts.

Nous avons calculé, pour chacune des équations, à partir de son écart type résiduel $\mathrm{S}$, un coefficient de variation résiduelle, C.V.R. $=\operatorname{I00} \times\left(\mathrm{I}-\mathrm{I} \mathrm{O}^{\mathrm{s}}\right)$, qui représente la variabilité résiduelle exprimée en pourcentage de $\mathrm{Y}$. Ce coefficient est un meilleur reflet de la liaison entre $\mathrm{Y}$ et $\mathrm{X}$ que le coefficient de corrélation, dépendant de l'étendue de variation de $\mathrm{X}$. 


\section{Résultats}

\section{A. - Résultats généraux}

Nous avons rapporté au tableau I, la moyenne des mesures les plus importantes correspondant aux quatre stades d'abattages. La vitesse de croissance moyenne des animaux est élevée ( $\mathrm{I} 226 \mathrm{~g} / \mathrm{j}$ ) et elle ne diminue que faiblement avec l'âge des animaux : I $367 \mathrm{~g} / \mathrm{i}(\sigma=229)$, I $2 \mathrm{Ig} \mathrm{g} / \mathrm{j}(\sigma=\mathrm{I} 29)$ et I $225 \mathrm{~g} / \mathrm{j}(\sigma=93)$ respectivement au cours des trois périodes $9-13,13-16$ et $16-19$ mois.

\section{TABIEAU I}

Poids moyen $(\mathrm{n}=5)$ des principaux éléments des animaux disséqués $(k g \pm \sigma)$ Mean weight of main components of dissected animals

\begin{tabular}{|c|c|c|c|c|}
\hline $\begin{array}{l}\text { Age (mois) } \\
\text { Age (month) }\end{array}$ & 9 & I 3 & 16 & 19 \\
\hline $\begin{array}{l}\text { Poids vif }(\text { body weight) } \\
\text { Poids vif vide }(e m p t y \text { body weight) } \\
\text { Carcasse }(\text { carctss) . . . . . . . . . . . . }\end{array}$ & $\left\{\begin{array}{l}304,5 \div 19,6 \\
260,5 \pm \mathrm{I} 5,8 \\
180,6 \pm 11, \mathrm{I}\end{array}\right.$ & $\begin{array}{l}440,4 \pm 24,3 \\
390,1 \pm 22,3 \\
271,4 \pm 17,0\end{array}$ & $\begin{array}{l}542,9 \pm 3 \mathrm{I}, \mathrm{O} \\
492,2 \pm 24,4 \\
348,0 \pm 17,2\end{array}$ & $\begin{array}{l}646,1 \pm 26,2 \\
585,5 \pm 23,2 \\
453,7 \pm 19,4\end{array}$ \\
\hline 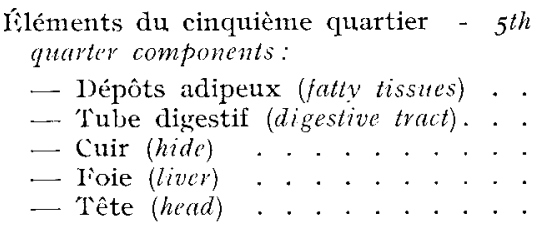 & $\begin{array}{r}3,9 \pm 0,3 \\
14,3 \pm 1,7 \\
24,3 \pm 2,7 \\
3,4 \pm 0,4 \\
8,8 \pm 0,7\end{array}$ & $\begin{array}{r}7,2 \pm 1,3 \\
19,6 \pm 1,9 \\
37,2 \pm 1,8 \\
5,2 \pm 0,5 \\
11,8 \pm 1,5\end{array}$ & $\begin{array}{r}10, I \pm 2,2 \\
19,5 \pm 1,5 \\
48,8 \pm 1,0 \\
5,1 \pm 0,3 \\
14,1 \pm 0,6\end{array}$ & $\begin{array}{r}17,3 \pm 9,3 \\
25,2 \pm 1,8 \\
53,8 \pm 4,2 \\
7,0 \pm 0,6 \\
15,9 \pm 0,4\end{array}$ \\
\hline 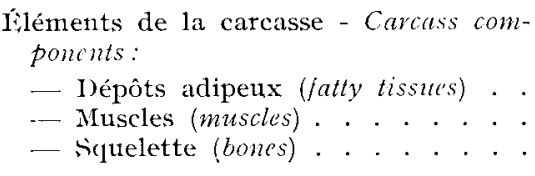 & $\begin{array}{r}15,3=1,4 \\
136,8 \pm 8,9 \\
27,2 \pm 1,9\end{array}$ & $\begin{array}{c}26,7 \pm 3,4 \\
207,5 \pm 13,9 \\
35,2 \pm 1,7\end{array} \mid$ & $\begin{array}{c}40,1 \pm 3,4 \\
263,7 \pm 12,6 \\
41,9 \pm 2,4\end{array}$ & $\begin{array}{c}54,9 \pm 5,3 \\
308,4 \pm 19,0 \\
48,4 \pm 1,3\end{array}$ \\
\hline $\begin{array}{l}\text { Dépôts adipeux de la carcasse - Carcass } \\
\text { fatty tissues: } \\
\text { - Internes (internal) . . . . . . . } \\
\text { — Intermusculaires (intermuscular) } \\
\text { - Sous-cutanés (subcutaneous) - . . }\end{array}$ & $\begin{array}{r}2,8 \pm I, 2 \\
I I, 2 \pm 2,2 \\
I, 3 \pm 0,4\end{array}$ & $\begin{array}{r}3,6 \pm 0,0 \\
20,0 \pm 2,9 \\
3,1 \pm 0,2\end{array}$ & $\begin{array}{r}5,8 \pm 1,0 \\
27,0 \pm 2,3 \\
6,0 \pm 1,8\end{array}$ & $\begin{array}{r}7,8 \pm 1,2 \\
37,5 \pm 0,3 \\
9,5 \pm 1,6\end{array}$ \\
\hline 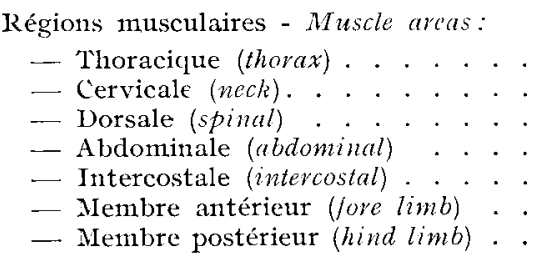 & $\begin{array}{r}13, \mathrm{I} \pm \mathrm{I}, 6 \\
\mathrm{I} 9,5 \pm \mathrm{I}, 7 \\
17,4 \pm \mathrm{I}, 4 \\
8,9 \pm 0,6 \\
6,5 \pm 0,5 \\
17,9 \pm 0,7 \\
57,3 \pm 5,0\end{array}$ & $\begin{array}{r}22,8 \pm 1,9 \\
33,3 \pm 5,0 \\
25,4 \pm 1,7 \\
13,7 \pm 0,7 \\
9,3 \pm 0,6 \\
27,1 \pm 2,0 \\
75,9 \pm 4,3\end{array}$ & $\begin{array}{l}30,5 \pm 2,0 \\
45,0 \pm 4,7 \\
32,2 \pm 2,0 \\
18,0 \pm 2,6 \\
12,1 \pm 1,0 \\
34,8 \pm 1,8 \\
91,0 \pm 2,9\end{array}$ & $\begin{array}{r}34,8 \pm 3,9 \\
5^{8,3} \pm 6,8 \\
35,4 \pm 1,5 \\
20,0 \pm 2,3 \\
15,0 \pm 0,5 \\
40,7 \pm 2,4 \\
104,5 \pm 7,2\end{array}$ \\
\hline
\end{tabular}


B. - Croissance relative des différents éléments du corps vide par rapport au poids vif vide (tab1. 2)

La carcasse a un coefficient d'allométrie supérieur à celui du cinquième quartier $(b=\mathrm{I}, 03$ contre 0,94$)$. Ce phénomène semble assez général chez les bovins puisqu'il a déjà été observé chez des bœufs de race Angus (SEEBEck, I967), chez des jeunes taureaux de race Frisonne (ROBELIN, GEAY et BÉRANGER, I974), de même que chez des bovins mâles entiers de race Rouge Danoise (ANDERSEN, I975).

\section{TABLEAU 2}

Croissance relative par rapport au poids vif vide $(X k g)$ des différents éléments $(Y k g)$ du corps entier (velative growth of various parts of the whole body)

$$
\log _{10} \mathrm{Y}=b_{0}+b_{1} \log _{10} \mathrm{X}+b_{2}\left(\log _{10} \mathrm{X}\right)^{2}
$$

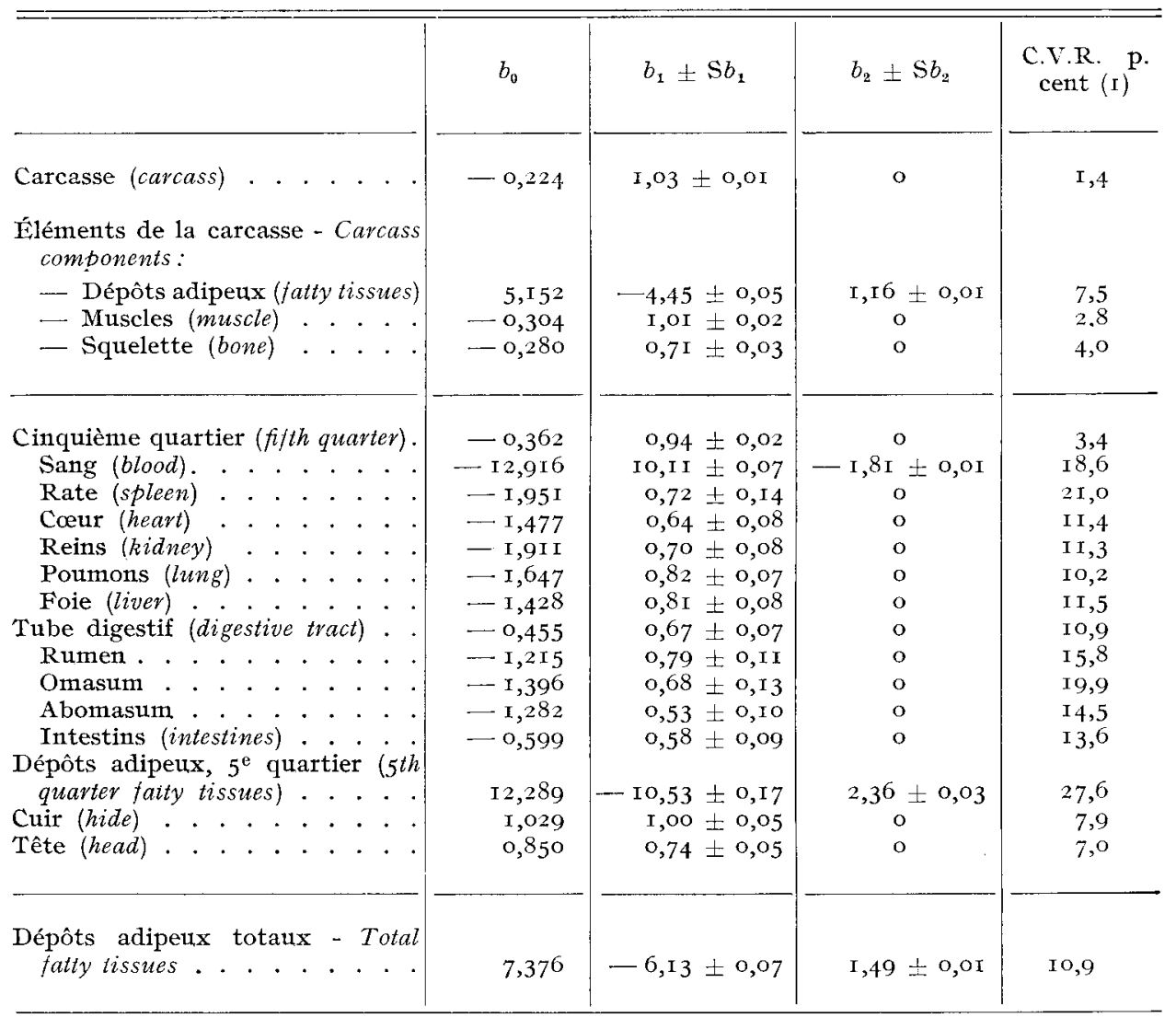

(I) C.V.R. = Coefficient de vatiation résiduel (écart type de Y exprimé en p. cent de $Y$ ). Residual variation coefficient (standard error of $Y$ expressed in $p$. cent of $Y$ ). 
Comme nous l'avions déjà observé chez les jeunes taureaux Frisons, la plupart des organes ont un coefficient d'allométrie inférieur à I (compris entre 0,5 et 0,9 ) . I, cuir a une croissance pratiquement isométrique $(b=\mathrm{I})$, plus rapide semble-t-il que chez les animaux de types plus précoces tels que les taurillons Frisons (RoBEIIN, Geay et BÉRANGer, I974) ou les bœufs Angus (SEEBECK, I967).

Nous retrouvons chez les taurillons Limousins la hiérarchie de croissance classique entre le squelette $(b=0,7 \mathrm{I})$, la musculature $(b=\mathrm{I}, \mathrm{OI})$ et l'ensemble des dépôts adipeux ( $b$ "moyen " $=\mathbf{I}, 59$ ).

Ces différentes croissances relatives ont pour conséquence, une augmentation lente du pourcentage de dépôts adipeux dans la carcasse $(8,5$ à I3,3 p. cent) ou dans le corps entier $(7,4$ à I 2,8 p. cent), une diminution lente du pourcentage du squelette dans la carcasse ( I 5 à II,7 p. cent) et une relative constance du pourcentage de muscles dans la carcasse $(75,8$ à 74,5 p. cent) (tabl. $3 \mathrm{~A}$ ).

I,e pourcentage de dépôts adipeux dans le gain de poids évolue plus rapide. ment, entre 12,6 et $22,5 \mathrm{p}$. cent dans le gain de poids de carcasse et entre II, 3 et 23,6 dans le gain de poids vif vide (tabl. $3 \mathrm{~B}$ ).

TABLEAU 3

A) Compositzon moyenne des animaux aux différents stades d'abattage

Mean body composition of slaughter groups animals

\begin{tabular}{|c|c|c|c|c|}
\hline Age (mois) - Age (months) & 9 & I3 & 16 & I9 \\
\hline Carcasse / poids vif vide (Carcass /Empty body weight) & 69,3 & 69,6 & 70,7 & 70,6 \\
\hline 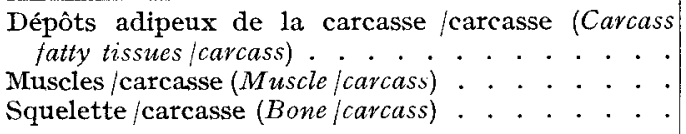 & $\begin{array}{r}8,5 \\
75,8 \\
\text { I } 5, \mathbf{I}\end{array}$ & $\begin{array}{r}9,8 \\
76,4 \\
13,0\end{array}$ & $\begin{array}{l}\text { I I }, 5 \\
75,8 \\
\text { I } 2,0\end{array}$ & $\begin{array}{l}\mathbf{1} 3,3 \\
74,5 \\
\mathbf{I}, 7\end{array}$ \\
\hline $\begin{array}{l}\text { Dépôts adipeux totaux/Poids vif vide (Total } \\
\text { fatty tissues/Empty body weight) } . . . \\
\end{array}$ & 7,4 & 8,7 & I0,2 & I 2,8 \\
\hline
\end{tabular}

B) Composition moyenne du gain de carcasse (p. cent) et du gain de poids vif vide ( $p$. cent) (Mean composition of carcass and empty body weight gain)

\begin{tabular}{|c|c|c|c|}
\hline $\begin{array}{l}\text { Période (months) } \\
\text { Period (months) }\end{array}$ & 9-I3 & I $3-16$ & I6-19 \\
\hline Gain de carcasse (Carcass gain) : & & & \\
\hline 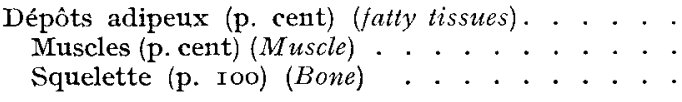 & $\begin{array}{r}12,6 \\
77,9 \\
8,8\end{array}$ & $\begin{array}{r}7,5 \\
73,4 \\
8,8\end{array}$ & $\begin{array}{r}22,5 \\
68,0 \\
9,9\end{array}$ \\
\hline $\begin{array}{l}\text { Gain de poids vif vide (Empty body weight gain) : } \\
\text { Dépôts adipeux (p. cent) (fatty tissues). . . . . . }\end{array}$ & I I, 3 & 16,0 & 23,6 \\
\hline
\end{tabular}




\section{C. - Croissance relative des différents types de dépôts adipeux}

La croissance relative de l'ensemble des dépôts adipeux (carcasse et $5^{\mathrm{e}}$ quartier) augmente par rapport à celle du poids vif vide entre 300 et $600 \mathrm{~kg}$ (tabl. $z$ ). Le coefficient $b$ passe de 1,25 à 2 , I5. L'amplitude de ce phénomène est pluss grande dans les dépôts du $5^{\text {e }}$ quartier ( $b$ augmente de I, I4 à 2,56$)$ que dans ceux de la carcasse ( $b$ augmente de I,30 à I,99).

Cette augmentation de la croissance relative des dépôts adipeux peut être caractérisée avec plus de précision grâce à la représentation de BARToN et LAIRD (I969) (Equation de GoMPERTZ). Selon ce mode d'expression, le taux de croissance du poids vif vide $\left(\frac{d \mathrm{P}}{\mathrm{P} d t}\right)$ diminue de $0,3 \mathrm{p}$. cent par jour chez les taurillons

\section{TABLEAU 4}

Croissance velative des différentes dépôts adipeux

(Relative growth of various fatty tissues)

$\left(\log \mathrm{Y}=b_{0}+b_{1} \log \mathrm{X}+b_{2}(\log \mathrm{X})^{2}\right)$

\begin{tabular}{|c|c|c|c|c|c|}
\hline $\mathrm{X}(\mathrm{kg})$ & Y (kg) & $b_{0}$ & $b_{1} \pm \mathbf{S} b_{1}$ & $b_{2} \pm \mathrm{S} b_{2}$ & $\begin{array}{l}\text { C.V.R. } \\
\text { p. cent }\end{array}$ \\
\hline $\begin{array}{c}\text { Poids vif vide } \\
\text { (Empty body } \\
\text { weight) }\end{array}$ & 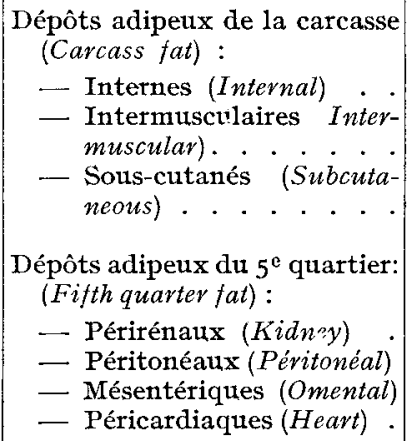 & $\begin{array}{l}-2,791 \\
-2,560 \\
-5,790 \\
\\
-4,397 \\
-4,304 \\
-3,562 \\
-2,760\end{array}$ & $\begin{array}{l}\mathrm{I}, 32 \pm 0,18 \\
\mathrm{I}, 49 \pm 0,07 \\
2,44 \pm 0, \mathrm{I} 7 \\
\\
\mathrm{I}, 8 \mathrm{r} \pm 0,2 \mathrm{I} \\
\mathrm{I}, 82 \pm 0,25 \\
\mathrm{I}, 48 \pm 0,2 \mathrm{I} \\
\mathrm{I}, 02 \pm 0,19\end{array}$ & $\begin{array}{l}0 \\
0 \\
0\end{array}$ & $\begin{array}{l}28,8 \\
10,8 \\
26,7\end{array}$ \\
\hline $\begin{array}{c}\text { Dépôt adipeux } \\
\text { inter musculaires } \\
\text { (Intermuscular } \\
\text { fat })\end{array}$ & $\begin{array}{l}\text { Dépôts du membre antérieur } \\
\text { (For limb IM fat) } \\
\text { Dépôts du tronc (Thoracic IM } \\
\text { fat) } \\
\text { Dépôts du membre postérieur } \\
\text { (Hind limb IM fat) . . . . }\end{array}$ & $\begin{array}{r}-\mathrm{I}, 032 \\
0,466 \\
-0,143\end{array}$ & $\begin{array}{l}\mathrm{I}, \mathbf{I} \mathbf{I} \pm 0,08 \\
\mathrm{I,I} 8 \pm 0,05 \\
0,66 \pm 0,05\end{array}$ & $\begin{array}{l}0 \\
0 \\
0\end{array}$ & $\begin{array}{l}9: 6 \\
6,0 \\
5,0\end{array}$ \\
\hline $\begin{array}{c}\text { Dépôts adipeux } \\
\text { sous-cutanés } \\
\text { (Subcutaneous } \\
\text { fat) }\end{array}$ & $\begin{array}{l}\text { Dépôts du membre antérieur } \\
\text { (For limb } S C \text { fat) } \\
\text { Dépôts du tronc (Thoraxic } S C \\
\text { fat) } \\
\text { Dépôts du membre postérieur } \\
\text { (Hind limb } S C \text { fat) }\end{array}$ & $\begin{array}{l}-0,889 \\
-0,34^{\circ} \\
-0,400\end{array}$ & $\begin{array}{l}0,88 \pm 0, \mathrm{I} 6 \\
\mathrm{I}, 08 \pm 0,05 \\
0,98 \pm 0,06\end{array}$ & $\begin{array}{l}0 \\
0 \\
0\end{array}$ & $\begin{array}{l}35,3 \\
\text { I0,8 } \\
\text { I } 2,4\end{array}$ \\
\hline
\end{tabular}


Limousins entre 9 et I 9 mois, alors que celui des dépôts adipeux ne diminue que de o,I p. cent par jour. C'est cette différence qui explique que la relation allométrique linéaire ne peut représenter qu'imparfaitement l'évolution du poids des dépôts adipeux en fonction du poids vif vide.

La croissance relative de la masse adipeuse n'est pas uniforme pour les différents dépôts (tabl. 4). Ce sont les dépôts adipeux sous-cutanés qui ont la croissance relative la plus forte $(b=2,44)$, suivis par les dépôts péritonéaux et périrénaux $(b \simeq \mathbf{r}, 8 \mathrm{o})$, puis par les dépôts intermusculaires $(b=\mathbf{1}, 49)$ et mésentériques $(b=\mathrm{I}, 48)$.

Au-delà des différences entre types de dépôts adipeux, il existe également des gradients de croissance à l'intérieur de certains dépôts selon leur localisation. Ainsi (tabl. 4), les dépôts intermusculaires et sous-cutanés ont une croissance relative beaucoup plus élevée au niveau du tronc ( $b=\mathrm{I}, \mathrm{I} 8$ et $\mathrm{x}, \mathrm{o} 8$ respectivement) que dans les membres. En particulier, la croissance relative des dépôts intermusculaires est très faible $(b=0,66)$ dans le membre postérieur.

\section{D. - Croissance relative des différentes régions musculaires par rapport à la musculature totale}

La croissance relative des muscles est très variable selon les régions corporelles (tab1. 5). Elle est supérieure à I,2 dans les régions cervicale et pectorale. En particulier, les muscles Rhomboïdeus, Trapezius et Cutanéus Troncï ont des coefficients d'allométrie très élevés (respectivement I,59; r,6o et I,30).

Les régions abdominale, intercostale et le membre antérieur ont une croissance relative voisine de celle de la musculature totale.

Enfin, la région dorsale et le membre postérieur ont des croissances relatives plus faibles que l'ensemble de la musculature ( $b$ compris entre 0,8 et 0,9$)$. De plus, dans le cas particulier du muscle psoas, elle diminue significativement entre 9 et I9 mois de 1,05 à 0,65 .

\section{$\mathrm{E}$. - Croissance relative des différentes parties du squelette}

Schématiquement, nous observons un gradient de croissance partant des extrémités des membres vers les ceintures : $0,78,0,80$, r, 08 respectivement pour le radius, l'humérus et l'omoplate dans le cas du membre antérieur : $0,76,0,80$, I,I6 respectivement pour le tibia, le fémur et le bassin dans le cas du membre postérieur. C'est au niveau du tronc que la croissance relative est la plus élevée ( $b=\mathrm{I}, 24$ dans le cas des vertèbres).

\section{F. - Variabilité du poids des différents éléments du corps vide}

A même valeur du poids vif vide, la variabilité du poids des différents éléments est généralement comprise entre ro et $20 \mathrm{p}$. cent de ce poids (tab1. 2). Elle atteint 30 à $40 \mathrm{p}$. cent pour les différents dépôts adipeux, mais seulement Io,9 p. cent pour l'ensemble de ces dépôts. Elle est beaucoup plus faible pour la musculature totale $(2,8$ p. cent) qui représente la moitié đu corps vide. A même poids de muscles totaux, la variabilité du poids des différentes régions musculaires est comprise entre 2,8 et 8,8 p. cent (tabl. 5). 
TABLEAU 5

Croissance relative des différentes parties de la musculature $(\mathrm{Y}, k g)$ par rapport

à l'ensemble de la musculatuve $(X, k g)$

Relative growth of various parts of musculature

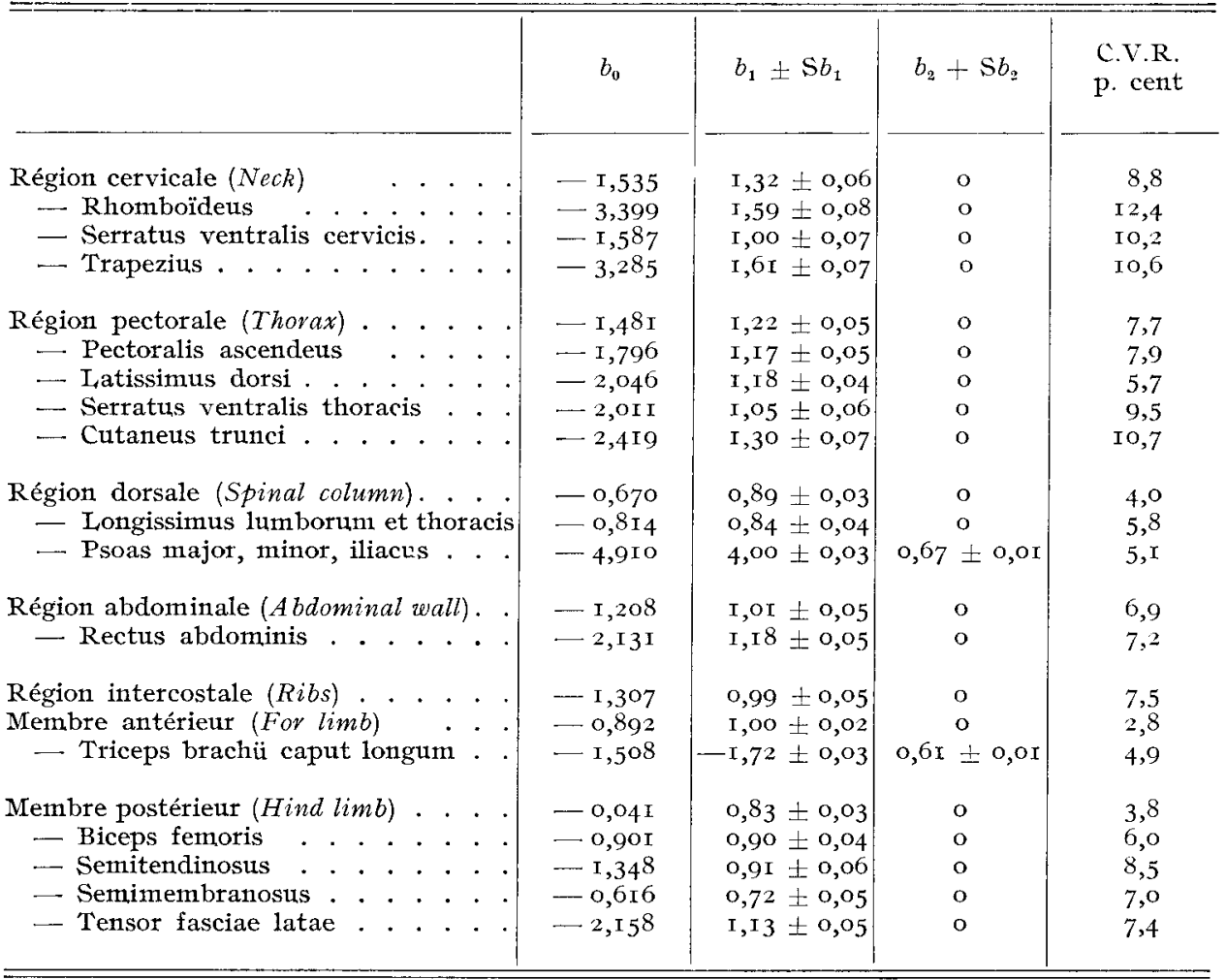

\section{Discussion}

Un certain nombre de conclusions importantes se dégagent de ces résultats, tant sur le plan général de la croissance de bovins, que sur les particularités des jeunes bovins mâles de race Limousine :

I) La relation d'allométrie linéaire ne semble pas permettre de décrire parfaitement l'évolution des dépôts adipeux, dont la croissance relative s'accélère au cours de la période que nous avons étudiée. Ce phénomène est assez général puisqu'on peut l'observer également dans les résultats de Schulz, OsLAGE et DAENICKE (I974) et que CANTIER et al. (I969) l'ont déjà mis en évidence chez les lapins. Ces derniers auteurs n'ont pas observé une évolution continue de $b$, mais plutôt un changement brusque d'allométrie. Tous ces résultats sont cependart très cohérents; il semble qu'après un stade physiologique que l'on peut situer proche de la puberté (environ $35^{\circ} \mathrm{kg}$ dans le cas des taurillons Limousins), durant lequel la croissance musculaire est maximale (AlMQUIST et al., I97I; ANDERSEN, 
I975), on observe une diminution de la vitesse de croissance relative des muscles au profit d'une augmentation de la croissance de la masse adipeuse.

2) Après les premiers résultats que nous avons obtenus sur des taurillons de race Frisonne (ROBEL 1 , GEAY et BÉRANGER, I974) puis de races Charolaise et Limousine (ROBELIN et GEAY, I976), il apparaît clairement maintenant que la croissance musculaire est beaucoup plus rapide dans les régions antérieures de l'animal (régions pectorale et cervicale) que dans la région dorsale et le membre postérieur. Il semble par ailleurs que ce sont les muscles plats ou superficiels (Rhomboïdus, Trapezius, Pectoralis Ascendeus, Cutaneus Trunci, Rectus Abdominis, Tensor Faciae Latae) qui présentent la plus forte croissance relative. Enfin, il est curieux de constater que les muscles dont la croissance relative est la plus faible sont généralement classés parmi les muscles les plus tendres (I ongissimus Dorsi, Psoas).

L'ensemble de ces résultats concorde parfaitement avec ceux qu'ANDERSEN (I975) a observés sur des taurillons Rouge Danois. Toutefois, à même poids de muscles dans la demi-carcasse (roo kg), les taurillons Limousins comportent plus de muscles dans la région dorsale et le membre postérieur que les taurillons Rouge Danois $(48,9$ contre $43, \mathrm{I} \mathrm{kg})$. D'après cette constatation et les résultats préliminaires que nous avons obtenus avec des taurillons de races Frisonne et Charolaise, il semblerait que la variabilité entre races, dans la répartition des masses musculaires, ne serait pas négligeable, et en tout cas bien supérieure à celle observée par BERG et BUTTERFIEI,D (I976).

3) La hiérarchie dans le développement des différents dépôts adipeux que nous avons observée semble assez générale chez les différents types de bovins d'après les résultats comparatifs que nous avons rapportés au tableau 6 . Toutefois, la croissance relative très élevée des dépôts périrénaux et péritonéaux ( $b$ voisin de $I, 8)$ peut paraître surprenante. Elle correspond à la valeur que nous avons observée par ailleurs (ROBELIN, GEAY et BÉRANGER, I974) chez des taurillons Frisons, à celle observée chez les ovins par Fourie, KirTon et JURY (I970) et Vezinheit et Prud'hon (I975) sur les dépôts péritonéaux. Ainsi, ces dépôts, dont le développement est très précoce (nous avons déjà pu les observer sur des foetus de bovins âgés de 5 mois et pesant $4 \mathrm{~kg}$ ), ont encore une croissance relative très élevée à un stade physiologique relativement avancé.

4) Les taurillons de race Limousine présentent des particularités dont une des plus importantes sur le plan pratique est leur rendement en carcasse (carcasse / poids vif vide) très élevé, de 69,3 à 70,6 p. cent contre 68 p. Ioo chez les taurillons Charolais et $64 \mathrm{p}$. cent chez les taurillons Frisons (GEAY et MALTERRE, 1973).

Par ailleurs, le coefficient d'allométrie des dépôts adipeux est bien inférieur aux valeurs obtenues précédemment $(I, 8$ à 2,4$)$ avec des taurillons de races laitières ou des animaux castrés de race Angus (ROBEL,IN, GEAy et BÉRANGER, I974; ANDERSEN, I975; SEEBECK et TUlioH, I968). Corrélativement, la croissance relative des muscles est plus élevée $(b=\mathrm{I}, \mathrm{OI})$ que chez ces animaux $(b=0,79$ à $0,98)$.

Enfin, les taurillons Limousins comportent pour un poids de carcasse de $220 \mathrm{~kg}$ (fig. I) Io à $\mathrm{I} 6 \mathrm{~kg}$ de dépôts adipeux de moins et $\mathrm{I} 3$ à $\mathrm{I} 5 \mathrm{~kg}$ de muscles de plus que les taurillons Frisons (SChUiz, OSLAGE et DAEnICKe, I974; Robelin, Geay et Beranger, I974) ou Rouge Danois (ANDERsen, I975). Comparés aux bœufs de race Angus, les taurillons Limousins comportent $40 \mathrm{~kg}$ de dépôts adipeux en moins pour un même poids de carcasse (fig. I).

Ces différences, jointes aux variations des coefficients d'allométrie, entraînent 
des écarts considérables dans la composition du gain de poids de carcasse, et surtout dans la proportion de dépôts adipeux. Entre I 80 et $270 \mathrm{~kg}$ de carcasse, le $\mathrm{kg}$ de gain de carcasse comporte I $26 \mathrm{~g}$ de dépôts adipeux chez les taurillons Limousins contre 230 à 3 Io g chez les taurillons Frisons ou Rouge Danois (ROBELIN, GEAy et BérAnger, 1974; ANDERSEN, 1975 (tabl. 3b).

Cette estimation des différences de potentiel de croissance entre animaux de races différentes, basée sur une comparaison à des résultats bibliographiques, peut être légèrement biaisée par des variations dans les conditions expérimentales. Toutefois, l'importance de ces différences, notamment par rapport à la variabilité
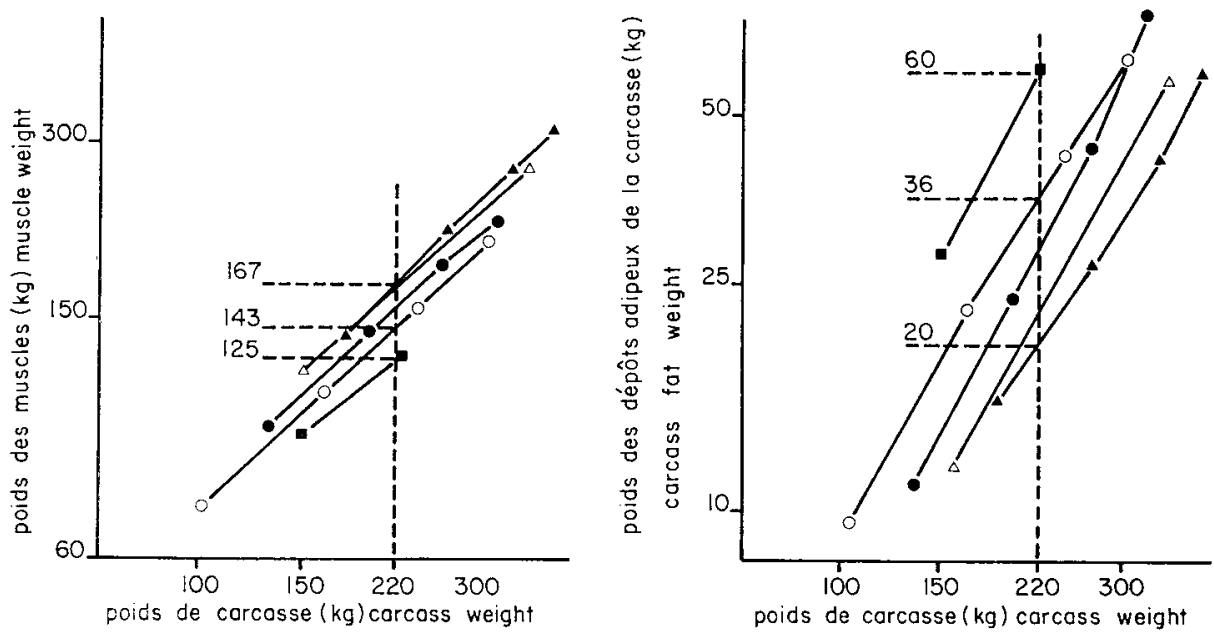

FIG. I. - Variabilité de la composition de la carcasse des bovins selon la vace et le sexe. $V$ ariability of carcass composition of cattle according to bred and sex.

- SEEBECK et TUl,LH (1968)

(Bœufs Angus - Angus steers)

ANDERSEN (1975)

(Taureaux rouge danois - Red Danish bulls)

- Schulz et al. (I974)

(Taureaux Frisons - Frisian bulls)

RoBELIN et al. (résultats non publiés - unpublished results)

(Taureaux Charolais - Charolais bulls)

Résultats publiés dans le texte - Results published in text

(Taureaux Limousins - Limousin bulls)

de la composition des animaux à même poids de carcasse (C.V.R. $=$ ro p. cent pour les dépôts adipeux), ne peut que refléter des différences réelles, liées au génotype de ces animaux. Ces différences ont une influence sur la valeur commerciale des carcasses; elles entraînent aussi des écarts importants dans le coût énergétique de production de ces carcasses; ce dernier point sera discuté sur la base de la composition chimique des animaux.

En plus des différences dans la quantité totale de dépôts adipeux existent également des différences dans la répartition de ces dépôts selon les types de bovins (tab1. 6). Pour un même poids de gras dans la carcasse (40 kg), les taurillons 


\section{TABI, EAU 6}

Croissance velative et répartition des différents types de dépôts adipeux chez les bovins Relative growth and distribution of bovine fatty tissues

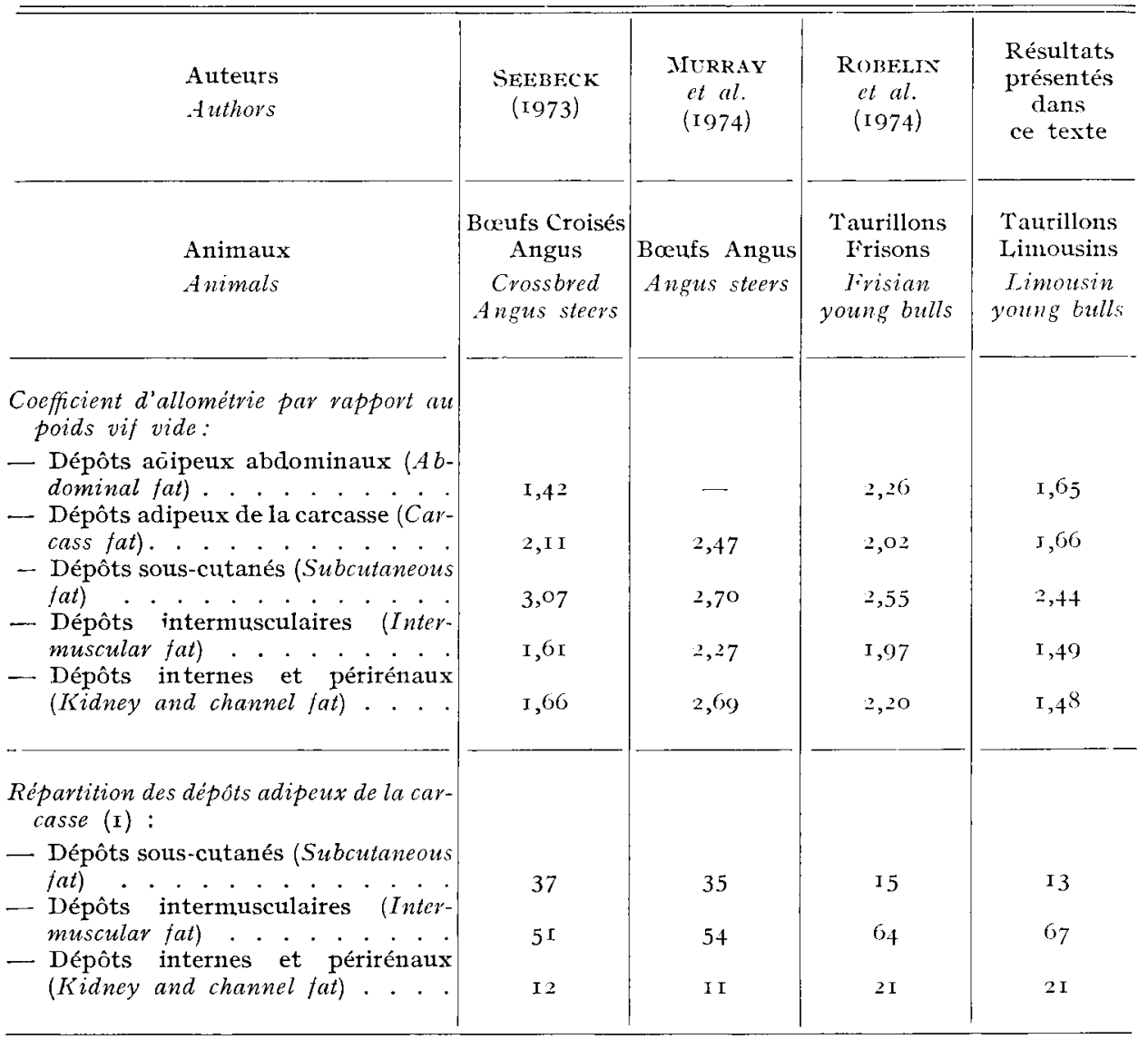

(r) Répartition des dépôts adipeux (en p. cent) pour un poids total de dépôts adipeux dans la carcasse de $40 \mathrm{~kg}$. Distribution of fat percentage in relation to total carcass fat weight (4o $\mathrm{kg}$.

Limousins comportent moins de dépôts sous-cutanés ( 13 p. cent des dépôts adipeux de la carcasse) que les taurillons Frisons (I5 p. cent), mais surtout beaucoup moins que les bœufs Angus (35 à $37 \mathrm{p}$. cent). Ces différences sont inversées au niveau des dépôts internes et périrénaux, mais surtout au niveau des dépôts intermusculaires. Une grande partie de ces différences (entre taurillons et boufs) est probablement liée à la castration, mais une certaine part doit être due au génotype de ces animaux. En effet, CHARLEs et Johnson (1976) ont observé également des différences très importantes entre des bœufs Angus, Hereford, Frisons et Croisés Charolais. Il est probable que ces différences, dans l'importance du gras sous-cutané, sont surtout le résultat de la sélection des animaux. En Firance, 
les animaux de races à viande ont été sélectionnés essentiellement sur l'importance de la masse musculaire, alors que dans les pays Anglosaxons, la sélection tendait surtout à favoriser l'augmentation de l'état d'engraissement, dont le reflet extérieur est 1'épaisseur du gras sous-cutané.

Les taurillons de race Limousine sont donc caractérisés par un faible état d'engraissement, notamment au niveau du gras sous-cutané, et un pourcentage de muscle élevé. Par ailleurs, ils renferment une quantité de muscles dans les régions dorsale et postérieure plus élevée que les animaux de races laitières.

La faible quantité de dépôts adipeux dans le gain de poids entraîne, sur le plan alimentaire, des besoins pour la croissance plus faibles que chez les animaux plus précoces (JARRIGE et al., r970). 'Toutefois, ce point pourra plus largement être discuté après l'étude de l'évolution de la composition chimique de ces animaux.

Accepté pour publication en juin 1977 .

\section{Summary \\ Evolution of body composition in young Limousine bulls \\ between 9 and Ig months of age}

The evolution of body composition in young Limousine bulls between 9 and 19 months of age is described. Five animals were slaughtered at $304,440,543$ and $646 \mathrm{~kg}$ body weight. The weight of offals, carcass, various fatty tissues, total muscles and various parts of musculature and bone were measured after dissection (Table I). Evolution of body composition is expressed in terms of allometric relationship of different measurements with empty body weight.

Classical patterns of growth (Table 2 ) were found for the skeleton $(b=0.7 \mathrm{I})$, musculature $(b=$ I.OI) and fatty tissues ( $b$ "mean $"=$ r.59). However, the grcwtl of fat deposits was better described by the quadratic relationship of logaritlim of body weight; the relative growth increased from 1.25 to 2.15 between 300 and $650 \mathrm{~kg}$ body weight.

Subcutaneous fat showed the highest relative growth $(b=2.44)$, followed by kidney and peritoneal fat $(b=1.80)$ then by intermuscular and omental fat $(b=1.48)$ ('Table 4$)$.

Muscles located along the spinal cord and in the hind limb showed the lowest relative growth, 0.89 and 0.83 respectively (Table 5). This result was already found with Friesian males (Rolikis et al., 1974) or Red Danish males (AxDERsis, 1975).

Some particularity of Limousine male cattle are discussed and mainly theirfat content. At the same carcass weight ( $220 \mathrm{~kg}$ ) (fig. I), Limousine cattle have io to $16 \mathrm{~kg}$ less fat than Friesian or Red Danish entire male (Schelz et al., I974; ANDHRSEx, 1975) and $40 \mathrm{~kg}$ less than Angus steers (SFEnICK and TULI,OH, I968). At the same fatty tissue weight (40 kg), Limousine male cattle have less subcutaneous fat ( 3 p. cent of carcass fatty tissue weight) than Friesian males (I 5 p. cent) or A1igus steers ( 35 to $37 \mathrm{p}$. cent) (Table 6). At the same muscle weight (Ioo kg in half carcass) Limousine male cattle lave more muscle located in the loin and the hind limb $(48.9 \mathrm{~kg})$ than Red Danish cattle (43.1 kg; ANDERSEN, 1975).

\section{Références bibliographiques}

Almquist C. N., Brungardt V. H., Tyler W. J, Waldman R. C., I97 I. Growth and efficiency of Holstein steers as infuenced by live weight and energy intake. J. Dairy Sci., 54, 6iI-687.

ANDERSEN H. R., I975. The influence of slaughter weight and level of feeding on growth rate, feed conversion and carcass composition of bulls. Liv. Prod. Sci., 2, 34 I-355.

ANONYME., I966. A comparison of the growtl of different types of cattle for beef production. Report of Major ISeef Research Project. The Royal Smithfield Club, London. 
BARTON A. D, IAARD A. K, I969. Analysis of allometric and non allometric differential growth. Growth, 33, I-I6.

BENEVENT M., I971. Croissance relative pondérale postnatale, dans les deux sexes des principaux tissus et organes de l'agneau Mérinos d'Arles. Ann. Biol. anim. Bioch. Biophys., 11, 5-39.

BE,RG R. T., BUTTERFIELD R. M., I976. Muscle growth patterns in steers. In "New concepts of cattle growth". Sydney press university p. 65-I42.

BUTTERFIELD R. M., I963. Relative growth of the musculature of the ox. In "Carcass composition and appraisal of meat animals", 7, X-I4. (Ed. D.E. TRIBE). East Melbourne. C.S.I.R.O.

CALLOW E. H., r948. Comparative studies of meat. II. The changes in the carcass during growth and fattening and their relation to the chemical composition of the fatty and muscular tissues. J. Agric. Sci., 38, I74-199.

Cantier J., Vezihnet A., Dulor J. P., Rouvier R., Dauzier L., ig69. Allométrie de croissance chez le lapin. IV. Principaux organes et tissus. Ann. Biol. anim. Bioch. Biophys., 9, 5-39.

Charless D. D., Johnson E. R., 1976. Breed differences in amount and distribution of bovine carcass dissectible fat. J. Anim. Sci., 42, 332-34I.

FourIE, P. D., KIRTON A. H., JURY K. E., I97o. Growth and development of sheep. II. Effect of breed and sex on the growth and carcass composition of the Southdown and Romney and their cross. N. Z. Journal Agric. Res., 13, 753-770.

GeAY Y., MalTerRe C., 1973. Ctoissance, rendement et composition des carcasses de jeunes bovins de différences races. Bull.Tech. C.R.Z.V. Theix, 14, i7-zo.

Jarrige R., Beranger C., Geay Y., Grenet N., Malterre C., Robei.in J., r97o. Besoins énergétiques des jeunes bovins. In "La production de viande par les jeunes bovins", p. I69-I 84 . Ed. S.E.I. C.N.R.A. Versailles. Fitude $\mathrm{n}^{0} 46$.

RobELIN J., GEAY Y., I976. Note : Répartition des masses miusculaires chez le jeune bovin mâle entier, et son évolution au cours de la période d'engraissement entre 8-9 et I6-I7 mois. Ann. Zootech., 25, 273-279.

ROBEi,in J., GE,AY Y., BERANGER C., I974. Croissance relative des différents tissus, organes et régions corporelles des taurillons Frisons, durant la phase d'engraissement de 9 à is mois. Ann. Zootech., 23, 313-323.

Schulz E., Oslage H. J., DaEnicke R., I974. Untersuchungen uber die zusammensetzung der korpersubstanz sowie den stoff und energieansatz bei wachsenden mastbullen. Fortschritte in der Tierphysiologie und Tievernährung., 4.

SEEBECK R. M., I967. Developmental growth and body weight loss of cattle. I. Experimental design, body weight growth and the effects of developmental growth and body weight loss on the dressed carcass and the offal. Aust. J. Agric. Res., 18, I015-3I.

SEEBECK R. M., TULIOH N. M., I 968 . Developmental growth and body weight loss of cattle. II. Dissected components of the commercially dressed and jointed carcass. Aust. J. Agric. Res., 19, 477-95.

Trowbridge P. F., Moulton C. R., Haigh L. D., Igig. Composition of the beef animal and energy cost of fattening. Mo. Agr. Expt. Station, Research Bulletin, $\mathrm{n}^{\mathrm{o}} 3 \mathrm{o}$.

TULLOH N. M., I963. The carcass composition of sheep, cattle and pigs as function of body weight. In "Carcass composition and appraisal of meat animal", 5, I-I6. Ed. D.E. 'TRIBE. East Melbourne C.S.I.R.O.

VEZINET A., PRUD'HON M., 1975. Évolution of various adipose deposits in growing rabbits and sheep. Anim. Prod., 20, 363-37o. 